\title{
Analytical and Numerical Methods for Solving Partial Differential Equations and Integral Equations Arising in Physical Models 2014
}

\author{
Santanu Saha Ray, ${ }^{1}$ Rasajit K. Bera, ${ }^{2}$ Adem Kılıçman, ${ }^{3}$ Om P. Agrawal, ${ }^{4}$ and Yasir Khan ${ }^{5}$ \\ ${ }^{1}$ Department of Mathematics, National Institute of Technology, Rourkela 769008, India \\ ${ }^{2}$ Department of Science, National Institute of Technical Teachers' Training and Research, BK-301, Sector II, Salt Lake City, \\ Kolkata 700091, India \\ ${ }^{3}$ Department of Mathematics and Institute for Mathematical Research, Universiti Putra Malaysia (UPM), 43400 Serdang, \\ Selangor, Malaysia \\ ${ }^{4}$ Department of Mechanical Engineering and Energy Processes, Southern Illinois University, Carbondale, IL 62901, USA \\ ${ }^{5}$ Department of Mathematics, Zhejiang University, Hangzhou 310027, China \\ Correspondence should be addressed to Santanu Saha Ray; santanusaharay@yahoo.com
}

Received 2 March 2015; Accepted 2 March 2015

Copyright (C) 2015 Santanu Saha Ray et al. This is an open access article distributed under the Creative Commons Attribution License, which permits unrestricted use, distribution, and reproduction in any medium, provided the original work is properly cited.

Nowadays, partial differential equations (PDEs) have become a suitable tool for describing the natural phenomena of science and engineering models. Most of the phenomena arising in mathematical physics and engineering fields can be expressed by PDEs. Many engineering applications are simulated mathematically as PDEs with initial and boundary conditions. Most physical phenomena of fluid dynamics, gravitation, chemical reaction, dispersion, nonlinear optics, plasma physics, quantum mechanics, electricity, acoustics, solid mechanics, and many other models are controlled within their domain of validity by PDEs. Therefore, it becomes increasingly important to be familiar with all traditional and recently developed methods for solving PDEs and the implementations of these methods.

For many years the subject of functional equations has held a prominent place in the attention of mathematicians. In recent years this attention has been focused on a specific kind of functional equations, an integral equation, where the unknown function occurs under the integral sign. Such equations occur widely in diverse fields including continuum mechanics, potential theory, geophysics, electricity and magnetism, kinetic theory of gases, hereditary phenomena in physics and biology, renewal theory, quantum mechanics, radiation, optimization, optimal control systems, communication theory, mathematical economics, population genetics, queuing theory, medicine, mathematical problems of radiative equilibrium, the particle transport problems of astrophysics and reactor theory, acoustics, fluid mechanics, steady state heat conduction, fracture mechanics, and radiative heat transfer problems. They offer a powerful technique for solving a variety of practical problems.

This special issue is devoted to study the recent works in the above fields of partial differential equations and integral equations done by the leading researchers. Accordingly, various papers on partial differential equations and integral equations have been included in this special issue after completing a heedful, rigorous, and peer-review process. The issue contains eight research papers.

The $(2+1)$-dimensional nonlinear Schrödinger equations (NLS) are transformed into the standard $(1+1)$-dimension nonlinear Schrödinger equation by using appropriate transformation and then by using Exp-function method bisolitons and a series of breather solitons (rogue waves) solutions are obtained. Solutions of the $(2+1)$-dimensional nonlinear Schrödinger equations, which contain Akhmediev breather soliton, Ma breather soliton and Peregrine breather soliton 
are represented. Also, higher-order rational rogue wave solutions are obtained for $(2+1)$-dimensional NLS equations by using the similarity transformation on the solutions of the (1 +1)-dimensional NLS equation. Several arbitrary parameters are involved to generate abundant wave structures which greatly enriched the diversity of wave structures for the $(2+$ 1)-dimensional nonlinear Schrödinger equations.

By using the central difference, upwind, hybrid, powerlaw, and exponential scheme, the general transport equation has been investigated. In the solution, three different grid systems of $80 \times 100,160 \times 200$, and $320 \times 400$ from nodal points were used by the authors. The obtained results indicate that hybrid and power-law yield better results as compared to the other discretization methods.

Some explicit travelling wave solutions are presented to construct the exact solutions of nonlinear partial differential equations of mathematical physics. Applying Frobenius transformation to the coupled Burgers, combined Kortewegde Vries- (cKdV-) modified KdV (mKdV) and Schrödinger$\mathrm{KdV}$ equations can be written as bilinear ordinary differential equations and two travelling wave solutions are generated. The properties of the multiply travelling wave solutions are shown in figures. The solutions thus obtained are stable and are used to describe nonlinear interaction of travelling waves.

The authors have studied the influence of heat transfer on thin film flow of a reactive third order fluid with variable viscosity and slip boundary condition. The problem is formulated in the form of coupled nonlinear equations governing the flow together with appropriate boundary conditions. Approximate analytical solutions for velocity and temperature are obtained by using optimal homotopy asymptotic method (OHAM) and are compared with Adomian decomposition method (ADM) solutions. Both of these solutions are found identical as demonstrated in graphs and tables. The graphical results for embedded flow parameters are also shown by the authors.

The nonlinear density temperature variations in twodimensional nanofluid flow over heated vertical surface with a sinusoidal wall temperature are examined in detail. The model includes the effects of Brownian motion and thermophoresis. Two-dimensional momentum, heat, and mass transfer equations are transferred to nonlinear partial differential equations using the boundary layer approximation and solved numerically using spectral local linearization method. The effects of the governing parameters on the fluid properties as well as on the heat and nanomass transfer coefficients are determined and are shown graphically.

An efficient fourth-order numerical algorithm that combines the Padé approximation in space and fourth-order accurate Rosenbrock method in time is proposed to solve a nonlinear parabolic partial differential equation. The semidiscrete ordinary differential equation (ODE) system resulting from compact higher-order finite difference spatial discretization of the reaction-diffusion equation is highly stiff. Therefore, numerical time integration methods with stiff stability such as implicit Runge-Kutta methods and implicit multistep methods are required to solve the large scale stiff ODE system. In this work the authors construct a new fourthorder Rosenbrock method to solve the nonlinear parabolic partial differential equation supplemented with Dirichlet or Neumann boundary condition. Two numerical examples are solved to demonstrate the efficiency, stability, and accuracy of the proposed method.

The modeling of unidirectional propagation of long water waves in dispersive media is considered. The authors have derived the Korteweg-de Vries (KdV) and Benjamin-BonaMahony (BBM) equations from water wave models. By implementing the extended direct algebraic and modified sech-tanh methods, the authors have presented new traveling wave solutions of the KdV and BBM equations. The stability of the obtained traveling wave solutions are analyzed and discussed.

Newtonian fluids described by Navier Stokes equations are extensively studied in the literature for the past few decades. The unsteady free flow of a Casson fluid over an oscillating vertical plate with constant wall temperature has been studied. The Casson fluid model is used to distinguish the non-Newtonian fluid behaviour. The governing partial differential equations corresponding to the momentum and energy equations are transformed into linear ordinary differential equations by using nondimensional variables. The dimensionless governing equations are solved by using the Laplace transform technique. Expressions for shear stress in terms of skin-friction and the rate of heat transfer in terms of Nusselt number are also obtained. Numerical results of velocity and temperature profiles with various values of embedded flow parameters are shown graphically and their effects are discussed in detail.

At the present, the use of partial differential equations and integral equations are commonly encountered in the fields of science and engineering. This issue has addressed some efficient computational tools, recent trends, and developments regarding the analytical and numerical methods for the solutions of partial differential equations and integral equations arising in physical models. Eventually, it may be expected that the present special issue would certainly help to explore the researchers with their new arising problems and stimulate the efficiency and accuracy of the solution methods for those problems in use nowadays.

Santanu Saha Ray Rasajit K. Bera Adem Kılıçman Om P. Agrawal Yasir Khan 


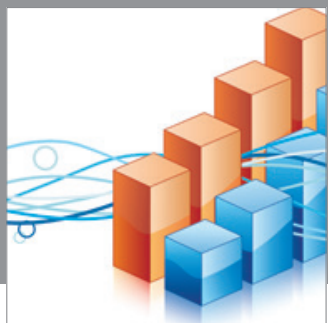

Advances in

Operations Research

mansans

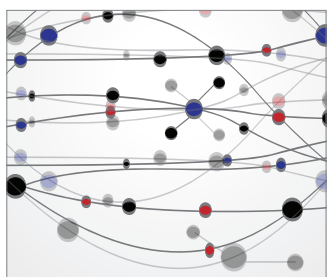

The Scientific World Journal
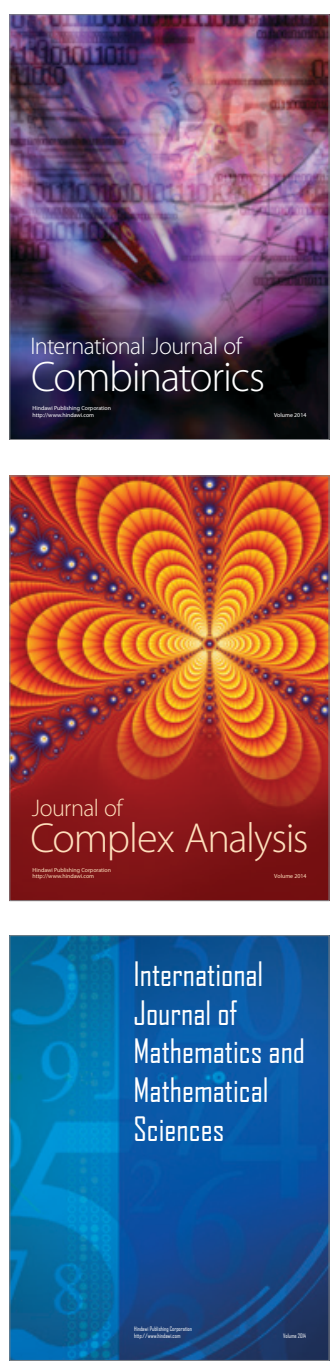
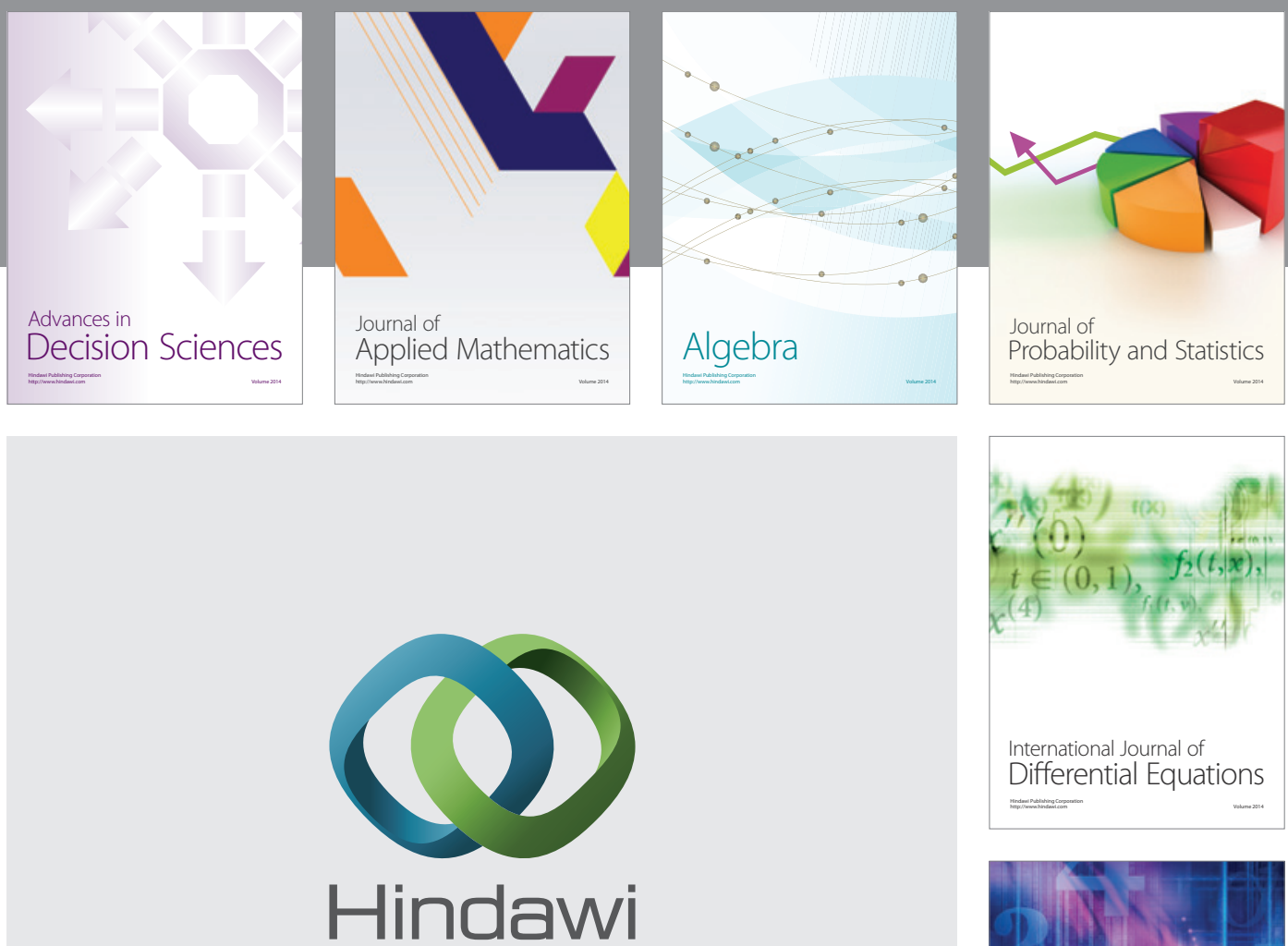

Submit your manuscripts at http://www.hindawi.com
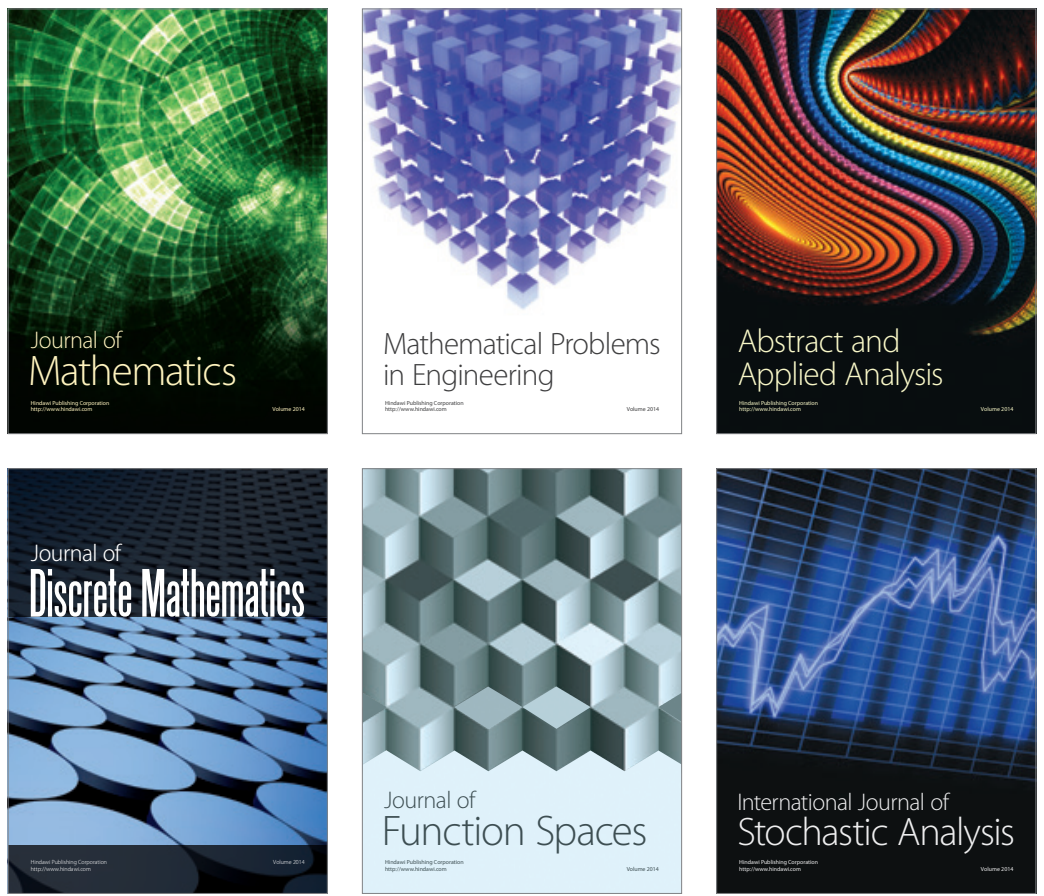

Journal of

Function Spaces

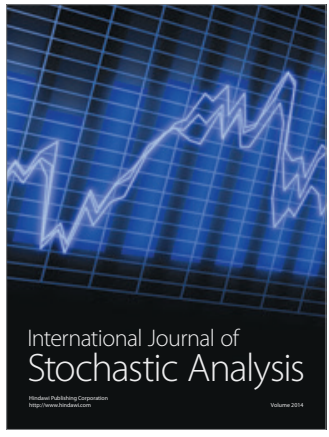

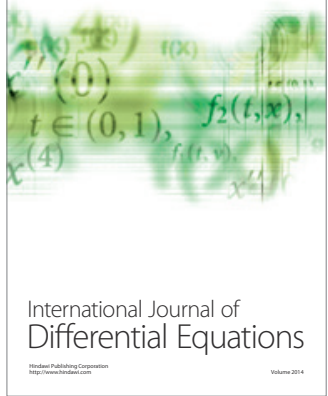
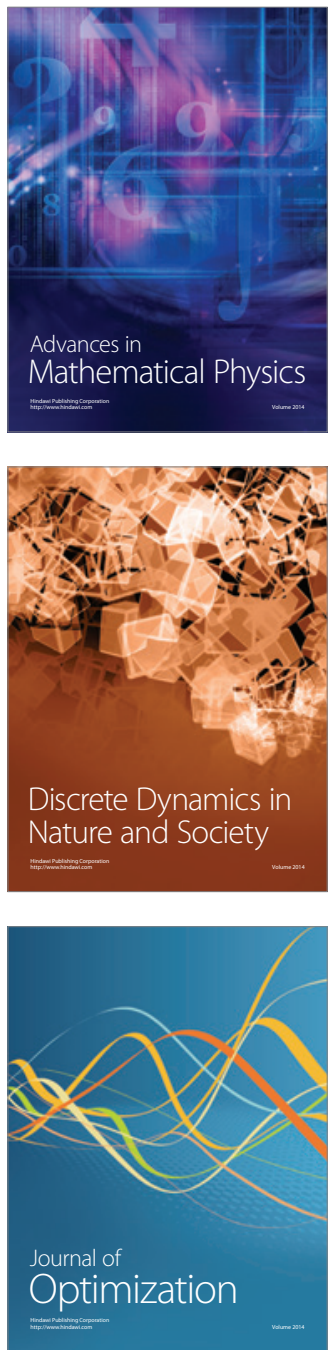